
Cahiers
de a Recherche
surles Droits
Fondamentaux

\section{Cahiers de la recherche sur les droits} fondamentaux

$13 \mid 2015$

Le droit d'asile

\title{
Les droits fondamentaux au carrefour de la Cour européenne des droits de l'homme et de la Cour de justice de l'Union européenne
}

Fundamental Rights at the Crossroads of the European Court of Human Rights and the European Court of Justice

\section{Carlos Ruiz Miguel}

\section{OpenEdition Journals}

Édition électronique

URL : http://journals.openedition.org/crdf/1254

DOI : $10.4000 /$ crdf. 1254

ISSN : 2264-1246

Éditeur

Presses universitaires de Caen

\section{Édition imprimée}

Date de publication : 1 novembre 2015

Pagination : 121-135

ISBN : 978-2-84133-742-2

ISSN : $1634-8842$

\section{Référence électronique}

Carlos Ruiz Miguel, « Les droits fondamentaux au carrefour de la Cour européenne des droits de I'homme et de la Cour de justice de l'Union européenne », Cahiers de la recherche sur les droits fondamentaux [En ligne], 13 | 2015, mis en ligne le 01 novembre 2016, consulté le 12 février 2020. URL : http://journals.openedition.org/crdf/1254; DOI : 10.4000/crdf.1254 


\title{
Les droits fondamentaux au carrefour de la Cour européenne des droits de l'homme et de la Cour de justice de l'Union européenne
}

\author{
Carlos RUIZ-MIGUEL \\ Professeur à l'université de Saint-Jacques de Compostelle (Espagne)
}

I. Les droits fondamentaux et les deux voies de la construction européenne

A. Un dessein européen ancien

B. La mise en place du Conseil de l'Europe

C. La "solidarité de fait» des Communautés européennes

D. L'incorporation progressive des droits fondamentaux dans la construction européenne

1. La promotion du processus d'unification politique par la jurisprudence de la Cour de Luxembourg

2. Une délicate incorporation des droits fondamentaux dans les traités européens

II. Les essais de convergence dans le domaine des droits de l'homme entre le Conseil de l'Europe et les Communautés européennes/Union européenne et la réticence de la Cour de justice de l'Union européenne
A. Les interférences entre les deux systèmes
B. Une prise en compte jurisprudentielle de la Convention européenne des droits de l'homme
C. L'exigence d'une compétence de l'Union en matière de droits fondamentaux
D. Un blocage persistant

III. La Charte des droits fondamentaux:

de l'essai de se passer du Conseil de l'Europe à la reconnaissance réticente de sa suprématie
$A$. La rédaction d'une charte inutile
B. Une concurrence impossible de la Convention européenne des droits de l'homme
C. Une alternative non viable

IV. Une convergence improbable: les difficultés et obstacles issus de l'avis de la Cour de justice de l'Union européenne du 18 décembre 2014
A. Des implications pour le Royaume-Uni
B. Un imparfait " amparo communautaire européen »
C. Une pertinente demande d'avis consultatif
D. Les conclusions de l'avis du 18 décembre 2014
E. Les véritables raisons du rejet 
L'Europe est un continent pionnier dans la protection internationale des droits de l'homme. C'est en Europe où, pour la première fois, une Cour a jugé des États suite à des plaintes d'individus pour violation des droits de l'homme. Cependant, le double processus d'intégration développé en Europe après la Seconde Guerre mondiale - l'un par le Conseil de l'Europe (CoE), l'autre par les Communautés européennes (aujourd'hui Union européenne - UE) pose la question de la protection juridictionnelle des droits de l'homme concernant les décisions de l'UE (I). Après l'échec de plusieurs essais de convergence entre les deux systèmes (II), le traité de Lisbonne de 2007 (en vigueur depuis décembre 2009) voulait ouvrir une voie pour régler ce problème avec une éventuelle adhésion de l'UE à la Convention de sauvegarde des droits de l'homme et des libertés fondamentales (Convention EDH), afin que la Cour européenne des droits de l'homme (Cour $\mathrm{EDH}$ ) puisse être saisie aux fins de vérifier le respect des droits de l'homme par les décisions de l'UE (III). Mais le chemin jusqu'à Lisbonne n'était pas facile et l'avis rendu par la Cour de justice de l'Union européenne (CJUE) le 18 décembre 2014 rend cette possibilité presque impossible (IV).

\section{Les droits fondamentaux et les deux voies de la construction européenne}

\section{A. Un dessein européen ancien}

Le désir d'une intégration européenne est certes très ancien, puisque l'on peut le dater de l'œuvre de Charlemagne. Mais les tentatives d'unification politique européenne sur une base libérale ou démocratique, avec une reconnaissance des droits fondamentaux, sont nettement plus récentes. On pourrait dire que la première expression d'un tel dessein se trouve dans le projet présenté par Saint-Simon et Thierry en 1814 après la défaite de Napoléon ${ }^{1}$. Saint-Simon propose alors une «Constitution» inspirée du modèle anglais ${ }^{2}$, avec un roi et un parlement bicaméral élu selon des critères censitaires et méritocratiques ${ }^{3}$, et garantissant certains droits comme la liberté de conscience et la liberté religieuse ${ }^{4}$. Ce projet ne fut pas retenu et la conférence de Vienne décida de soutenir l'instauration des monarchies absolues en Europe.

Il y eut bien ensuite quelques mouvements européistes de caractère libéral, mais le triomphe des absolutismes dans la première moitié du XIX ${ }^{e}$ siècle et l'ère des nationalismes et des impérialismes dans la seconde moitié du même siècle et au début du $\mathrm{XX}^{\mathrm{e}}$ siècle ont écarté de telles propositions.
Il faut attendre la fin de la Première Guerre mondiale pour que de tels projets soient réactivés. La guerre avait détruit l'Europe et deux grandes puissances qui menaçaient de l'éclipser avaient émergé à ses côtés: les ÉtatsUnis d'Amérique et l'Union des républiques socialistes soviétiques (URSS). C'est à ce moment que réapparaît le mouvement européiste libéral sous la conduite du comte Richard Coudenhove-Kalergi qui en 1923 publia un important livre pamphlet, Pan-Europa, incitant à la création d'un État fédéral européen suivant le modèle des États-Unis ${ }^{5}$. Dans une première phase, Coudenhove-Kalergi ne faisait pas référence dans son œuvre aux droits fondamentaux, mais seulement aux problèmes d'articulation entre plusieurs États souverains. Mais lorsqu'éclata la Seconde Guerre mondiale, Coudenhove-Kalergi livra un nouveau livre agonique L'Europe doit s'unir (Europe Must Unite) où l'on trouvait un manifeste («Appel à tous les Européens ») dans lequel il considérait que l'Europe n'avait pas de futur en suivant les chemins du bolchevisme ou du nationalisme et qu'elle ne pourrait survivre qu'en s'unissant. Dans cet appel il considérait que la Fédération des «États-Unis de l'Europe » devait s'organiser sur des bases libérales pour assurer, parmi d'autres objectifs:

L'obligation de tous les États européens, sans considération des différences de ses Constitutions, de respecter les droits de la personne humaine et l'égalité des citoyens appartenant à des minorités ethniques ou religieuses ${ }^{6}$.

\section{B. La mise en place du Conseil de l'Europe}

La fin de la Seconde Guerre mondiale aboutit à des destructions encore plus vastes que celle de la Première Guerre mondiale. Cette situation donna des forces aux mouvements européistes de type libéral-démocratique. En mai 1948, le Comité international des mouvements pour l'unité européenne organisa une conférence à La Haye. Lors de cette conférence, un rapport proposait l'idée de créer une "assemblée» pour donner une impulsion à l'UE (ce qui sera concrétisé par la création du $\mathrm{CoE}$ ) et celle d'imposer aux membres du futur $\mathrm{CoE}$ de souscrire à une déclaration des droits fondamentaux comme condition pour leur admission audit Conseil. Mais on ajoutait encore deux choses: primo, que les États devraient reconnaître à ce Conseil «le droit d'assurer ce respect» des droits fondamentaux; et secundo, que l'on instituerait une Cour européenne, indépendante, à laquelle «tout membre du Conseil pourrait soumettre les cas lors desquels la déclaration des droits semblait avoir été violée». Sur la base de ce rapport, la commission juridique du mouvement européen

1. C.-H. de Saint-Simon, A. Thierry, De la réorganisation de la société européenne, Paris, A. Égron, 1814.

2. Ibid., p. XV.

3. Ibid., p. $49 s q$

4. Ibid., p. 61 .

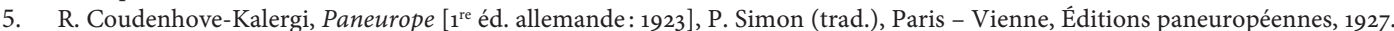

6. R. Coudenhove-Kalergi, Europe Must Unite, Glarus, Paneuropa Editions, 1939, p. 159 (nous traduisons). 
rédigea un premier projet de convention présenté à la conférence de Bruxelles de février $1949^{7}$.

L'«assemblée» prit forme avec la création du CoE lors de l'approbation du Statut du CoE à Londres par dix États européens ${ }^{8}$. Cependant, le CoE fut une grande frustration pour les espoirs européistes. Certes, on trouvait une rhétorique «fédéraliste» qui ressemblait à celle de la Constitution nord-américaine ${ }^{9}$ et l'annonce du renforcement de l'union entre les États par

[...] l'adoption d'une action commune dans les domaines économique, social, culturel, scientifique, juridique et administratif, ainsi que par la sauvegarde et le développement des droits de l'homme et des libertés fondamentales ${ }^{10}$.

Mais ces buts ambitieux ne trouvaient pas de concrétisation dans les articles du Statut: l' «assemblée» ne disposait pas de compétences de décision et ne pouvait qu'émettre des recommandations (art. 23 et 24 du Statut du CoE). Et last but not least, le Statut instituant le CoE ne contenait pas de référence à la Cour européenne indépendante demandée lors de la conférence de La Haye de 1948.

La grande frustration générée par les lacunes du Statut du CoE fit que l'on souhaita compenser les efforts et illusions des européistes par l'adoption, l'année suivante, de la Convention EDH. Ce texte établissait, pour la première fois, une déclaration européenne des droits de l'homme accompagnée d'un mécanisme international pour veiller à son respect ${ }^{11}$. Mais le mécanisme restait loin des aspirations des mouvances européistes. Certes, on établissait une Cour européenne des droits de l'homme, mais il s'agissait d'une cour « internationale» que seuls les États membres et un organisme international (la Commission européenne des droits de l'homme) pouvaient saisiri ${ }^{12}$.

\section{La "solidarité de fait" des Communautés européennes}

L'approbation de la Convention EDH représenta un nouvel échec partiel pour le mouvement européiste, parce que le texte restait éloigné de ce qu'avaient demandé les promoteurs de l'union politique européenne. Ce nouveau trauma donna un élan à l'initiative proposée par le ministre français des Affaires étrangères, l'Alsacien Robert Schuman, un an après l'approbation du Statut du CoE. Dans sa déclaration du 9 mai 1950, il disait:
«L'Europe ne se fera pas d'un coup, ni dans une construction d'ensemble: elle se fera par des réalisations concrètes créant d'abord une solidarité de fait».

Dans ladite déclaration Schuman, il proposa une première étape vers une «réalisation concrète» créant cette « solidarité de fait». Ainsi, recueillant une idée déjà exprimée par Coudenhove-Kalergi en 1923, il proposa de "placer l'ensemble de la production franco-allemande de charbon et d'acier sous une Haute Autorité commune, dans une organisation ouverte à la participation des autres pays d'Europe». Pour Schuman, ce serait «la première étape de la Fédération européenne, [qui] changera le destin de ces régions longtemps vouées à la fabrication des armes de guerre dont elles ont été les plus constantes victimes $»^{13}$.

L'appel de Schuman fut à l'origine de la Communauté européenne du charbon et de l'acier (CECA) créée à Paris l'année suivante (1951) par six États (l'Allemagne de l'Ouest et la France, en plus de l'Italie, de la Belgique, des Pays-Bas et du Luxembourg). Cinq États fondateurs du CoE (le Danemark, l'Irlande, la Norvège, le Royaume-Uni et la Suède) plutôt réticents à une démarche fédérale en Europe ne voulurent pas, à ce moment, y adhérer. Le traité CECA était purement économique et n'incluait pas d'articles de nature politique ou relatifs aux droits fondamentaux. La CECA déclencha un processus économique de construction européenne qui diffère du processus d'essence politique mis en place sous la direction du CoE. Apparaît ici une divergence dans les efforts mis en œuvre visant la création d'une unité européenne, encore plus frappante si l'on constate que les participants aux deux processus étaient différents.

Le succès de la CECA, créée à l'initiative de Schuman, fit encore croire aux fédéralistes européens qu'il était possible de parvenir à l'union politique européenne. On essaya à nouveau l'union politique mais cette fois avec seulement les six États engagés dans le processus de solidarité économique. Un an après le traité CECA, fut présenté, le 27 mai 1952, un projet de traité instituant une Communauté européenne de défense (CED). L'inspiration de Coudenhove-Kalergi et l'objectif européiste étaient tout à fait clairs. Le préambule du projet de traité CED affirmait d'un côté que les États signataires se montraient «soucieux de sauvegarder les valeurs spirituelles et morales qui sont le patrimoine commun de leurs peuples» et, de l'autre côté que les signataires étaient «conscients de

7. Voir M. Díez de Velasco y Vallejo, «Mecanismos de garantía y medios procesales de protección creados por la Convención Europea de los Derechos del Hombre», in Homenaje a D. Nicolás Pérez Serrano, Madrid, Reus, 1959, t. II, p. 585 sq., spéc. p. 586-587.

8. Statut du Conseil de l'Europe, signé à Londres, le 5 mai 1949. Les dix États fondateurs furent (par ordre alphabétique): la Belgique, le Danemark, la France, l'Irlande, l'Italie, le Luxembourg, la Norvège, les Pays-Bas, le Royaume-Uni et la Suède. La République fédérale d'Allemagne (l'Allemagne «occidentale») n'existait pas encore, parce que sa Loi fondamentale ne fut approuvée que quelques jours plus tard (le 23 mai 1949).

9. Le préambule prévoyait qu' "une union plus étroite s'impose entre les pays européens qu'animent les mêmes sentiments». De plus, selon l'article 1.a, «Le but du Conseil de l'Europe est de réaliser une union plus étroite entre ses membres afin de sauvegarder et de promouvoir les idéaux et les principes qui sont leur patrimoine commun et de favoriser leur progrès économique et social».

10. Art. 1.b du Statut du CoE.

11. Convention de sauvegarde des droits de l'homme et des libertés fondamentales, conclue à Rome, le 4 novembre 1950.

12. Ce n'est qu'en 1998 que les individus furent autorisés à saisir directement la Cour avec la réforme de la Convention opérée par le Protocole n 11 à la Convention de sauvegarde des droits de l'homme et des libertés fondamentales, signé le 11 mai 1994, et entré en vigueur en 1998.

13. On trouve le texte de la déclaration dans R. Schuman, Pour l'Europe, Paris, Nagel, 1963, p. 201 sq. 
franchir ainsi une étape nouvelle et essentielle dans la voie de la formation d'une Europe unie». La CED était une étape essentielle pour achever l'union politique. Et parce que l'un des aspects de toute union politique est la reconnaissance des droits fondamentaux, le projet de traité CED établit que cette Communauté

[...] ne recourt à des interventions que dans la mesure nécessaire à l'accomplissement de sa mission et en respectant les libertés publiques et les droits fondamentaux des individus ${ }^{14}$.

Cette reconnaissance des droits fondamentaux, pour la première fois dans le processus mené par les six fondateurs de la CECA, interférait avec le processus d'union politique parrainé par le CoE. Mais la porte restait ouverte pour une convergence entre les deux processus.

En étroite relation avec le projet de traité CED, une assemblée ad hoc, composée de l'Assemblée parlementaire de la CECA et des six États membres, adopta le 10 mars 1953 un projet de traité portant statut de la Communauté européenne (SCE). Ce projet prévoyait l'établissement d'une véritable communauté politique européenne avec une claire inspiration fédéraliste et contenait de nombreuses références aux droits fondamentaux. Dans son préambule, les signataires se montraient « décidés à sauvegarder ensemble la dignité, la liberté et l'égalité fondamentale des hommes, quelle que soit leur condition, leur race ou leur religion». Les articles du projet de traité SCE présentent de nombreuses et importantes références aux droits fondamentaux. Ainsi, l'article 2 établit que parmi les buts de la communauté politique européenne se trouve celui de «contribuer à la sauvegarde, dans les États membres, des droits de l'homme et des libertés fondamentales». L'article 3 va même au-delà en prévoyant que

Les dispositions du titre I de la Convention de sauvegarde des droits de l'homme et des libertés fondamentales, signée à Rome le 4 novembre 1950, ainsi que celles du protocole additionnel, signé à Paris le 20 mars 1952, sont parties intégrantes du présent Statut.

Il faut noter que le projet de SCE proposait l'incorporation de la déclaration des droits de la Convention $\mathrm{EDH}$ (titre I), mais non de son mécanisme de garantie (titre II). Ainsi, même si la Communauté européenne intégrait le catalogue des droits de la Convention EDH élaboré par le $\mathrm{CoE}$, elle restait en dehors de son mécanisme de garantie (alors composé par la Commission européenne des droits de l'homme et la Cour EDH). Une possible explication de cette situation pourrait être que seuls des États pouvaient adhérer à la Convention EDH et la Communauté n'était pas un État. En conséquence, la Communauté pouvait intégrer la Convention EDH au sein du droit communautaire, mais elle ne pouvait pas adhérer à la Convention EDH et, donc, ne pouvait se soumettre à la juridiction de la Cour EDH.
Cependant, les rêves fédéralistes européens allaient encore se heurter à la réalité du nationalisme politique. Le 30 août 1954, le Parlement français, sous la pression conjointe des gaullistes et des communistes, refusa d'adhérer au traité CED, auquel les cinq autres États membres de la CECA avaient déjà adhéré. L'échec de la CED comportait un gel immédiat du projet politique de SCE.

Le rejet du traité CED, la même année que la défaite à Diên Biên Phû, eut en France un effet tout à fait opposé aux intentions de la CED : celui d'accélérer la décision d'entreprendre toutes les démarches possibles pour se doter de l'arme atomique. Une décision que la France n'aurait pu adopter si la CED avait été approuvée alors que cette même décision semblait pressante pour l'Armée française depuis 1952, date à laquelle le Royaume-Uni avait réussi à se doter de sa propre arme atomique ${ }^{15}$.

La fracture dans le processus de construction européenne était alors effective. D’un côté, le CoE continuait à promouvoir l'intégration européenne sur la base fondamentale de la Convention EDH; de l'autre côté, les six États fondateurs de la CECA approfondissaient leur route vers l'union économique avec l'établissement en 1957 de deux nouvelles communautés essentiellement économiques : la Communauté économique européenne (CEE) et la Communauté européenne de l'énergie atomique (CEEA). Toutes les deux, comme la CECA, relevaient d'une approche strictement économique éludant toute référence à la reconnaissance des droits fondamentaux et à un contrôle international ou supranational du respect desdits droits.

\section{L'incorporation progressive des droits fondamentaux dans la construction européenne}

Le mécanisme de reconnaissance et de protection des droits fondamentaux établi par le CoE se consolida lentement mais d'une façon pérenne. Les Communautés européennes ne se cantonnèrent pas à être seulement une union économique et essayèrent, à travers diverses voies, de doter d'un caractère «politique» l'ordre juridique communautaire. Mais cette opération s'effectua de manière irrégulière (ultra vires) ou insuffisante (Acte unique européen, traité d'Union européenne). Le temps joua cependant contre cette stratégie. Pendant cette période où les Communautés européennes n'établirent pas les choses de façon appropriée, le CoE consolida en revanche sa position en tant que référence dans le domaine des droits de l'homme en Europe.

Les traités instituant les trois Communautés européennes ne faisaient pas allusion aux droits fondamentaux. Certes, ils contenaient des dispositions qui avaient des liens avec les droits fondamentaux (comme la liberté 
d'établissement ou la liberté de circulation), mais il ne s'agissait pas vraiment de "droits de l'homme», car il s'agissait seulement des droits des acteurs économiques. La personne qui n'évoluait pas comme homo aeconomicus ne jouissait pas des droits d'après les traités communautaires. Il était clair qu'une telle situation ne pourrait être surmontée qu'avec une réforme des traités qui, d'un côté, pourrait reconnaître des «droits fondamentaux» (donc attribués à toutes les personnes et pas seulement aux acteurs économiques) et, d'un autre côté, pourrait accorder à ces droits le même mécanisme de protection que les traités offrent aux autres dispositions.

\section{La promotion du processus d'unification politique \\ par la jurisprudence de la Cour de Luxembourg}

La Cour de justice des Communautés européennes (CJCE) voulait arracher sur le terrain judiciaire ce que le mouvement européiste n'avait pas obtenu des Parlements ni des gouvernements. La construction du droit communautaire par la CJCE, jusque-là qualifiée de "prétorienne» (même si le qualificatif apparaît inexact), s'est articulée autour de deux axes: le premier, que les traités font naître directement des droits au profit des particuliers qui peuvent être invoqués devant les cours nationales; le second, que le droit communautaire prévaut sur le droit national dans le cas d'un conflit entre les deux. La première idée (les traités génèrent directement des droits et des obligations pas seulement pour les États membres, mais aussi pour les citoyens) fut exprimée dans l'arrêt Van Gend \& Loos de $1963^{16}$. La deuxième (le droit issu des traités communautaires prévaut sur le droit national de chaque État) est contenue dans l'arrêt Costa de $1964^{17}$. L'articulation de ces deux idées signifiait un essor spectaculaire pour le processus de construction politique européenne.

Mais c'était trop tard. Le 13 février 1960 explosait la première bombe nucléaire française. À partir de cet événement, il était pratiquement impossible d'aboutir à une véritable union politique parce qu'il était tout à fait improbable que la France soit disposée à céder à une éventuelle union politique européenne le contrôle sur ses armes nucléaires. En plus de ces insurmontables difficultés politiques existaient d'autres obstacles.

Sans attendre que mûrisse cette "solidarité de fait» dont parlait Schuman, la Cour de justice avait forcé la marche vers l'union politique européenne suivant le modèle nord-américain, où la construction du fédéralisme doit beaucoup au rôle joué par la Cour suprême. Aux États-Unis, cette Cour avait d'abord affirmé que la Constitution fédérale était une norme obligatoire qui faisait directement naître des droits et des obligations pas seulement pour les États fédérés, mais aussi pour les citoyens; elle avait ensuite affirmé que le droit de la Constitution fédérale était supérieur à celui des États fédérés. À partir de ces raisonnements, et en s'appuyant sur une interprétation extensive des compétences constitutionnelles de la Fédération (comme la clause de commerce), on assura la construction fédérale de l'union politique nord-américaine.

Cependant, la Cour de justice européenne oubliait les différences importantes entre les cas européen et nordaméricain: l'homogénéité sociale, politique et culturelle aux États-Unis était supérieure à celle de l'Europe; en outre, le fédéralisme s'était imposé avec la défaite militaire des anti-fédéralistes dans une guerre sanglante. Mais existait encore une autre différence : la Constitution fédérale comme celles des États américains fédérés contenaient des déclarations reconnaissant des droits fondamentaux aux individus. Cependant, en Europe les droits fondamentaux ne se trouvaient pas dans les textes du processus d'intégration déclenché par la CECA, mais se trouvaient seulement dans les Constitutions des États membres de la CECA et dans le texte instituant l'autre voie vers l'intégration politique : la Convention EDH du CoE. Weiler a bien noté que le talon d'Achille de l'expansion du droit communautaire se trouvait ici: comment justifier la prévalence d'un droit qui ne consacre pas les droits fondamentaux (le communautaire) sur celui qui le fait (l'étatique ${ }^{18}$ ?

La Cour de justice guidée par un élan de «despotisme éclairé » essayait à tout prix de promouvoir un processus d'union politique du sommet à la base comblant ainsi le déficit des droits fondamentaux dans un processus pénible ${ }^{19}$ dont voici les étapes marquantes:

Dans une première phase, on affirma que «les droits fondamentaux de la personne» étaient «compris dans les principes généraux du droit communautaire dont la Cour assure le respect ${ }^{20}$.

Peu après ${ }^{21}$, la Cour combina cette doctrine avec celles de l'effet direct et de la primauté, ce qui suscita de grandes craintes dans plusieurs pays européens ${ }^{22}$.

16. Arrêt CJCE, 5 février 1963, NV Algemene Transport en Expeditie Onderneming van Gend \& Loos c. administration fiscale néerlandaise, C-26/62, Recueil, p. 1.

17. Arrêt CJCE, 15 juillet 1964, Flaminio Costa c. E.N.E.L., C-6/64, Recueil, p. 1141

18. J. H. H. Weiler, "The Jurisprudence of Human Rights in the European Union. Integration and Disintegration, Values and Processes», Jean Monnet Working Paper, $\mathrm{n}^{\circ}$ 2/96, en ligne: http://jeanmonnetprogram.org/archive/papers/96/9602.html.

19. Pour une analyse plus détaillée de ce processus, voir C. Ruiz-Miguel, «El largo y tortuoso camino hacia la Carta de los Derechos Fundamentales de la Unión Europea», in Estudios sobre la Carta de los derechos fundamentales de la Unión Europea, C. Ruiz-Miguel (dir.), Saint-Jacques de Compostelle, Université Saint-Jacques de Compostelle, 2004, p. 13 sq., spéc. 26 sq.

20. Arrêt CJCE, 12 novembre 1969, Erich Stauder c. ville d'Ulm - Sozialamt, C-29/69, Recueil, p. 419.

21. Arrêt CJCE, 17 décembre 1970, Internationale Handelsgesellschaft mbH c. Einfuhr- und Vorratsstelle für Getreide und Futtermittel, C-11/70, Recueil, p. 1125 .

22. Arrêt de la Cour constitutionnelle italienne, 27 décembre 1973, Frontini et autres c. ministère des Finances, Giurisprudenza Costituzionale, $\mathrm{n}^{\circ}$ 183/1973, t. I, p. 240 sq.; ordonnance (Beschluss) de la Cour constitutionnelle fédérale allemande, 29 mai 1974, Solange, BverfGE, t. 37, p. 271 sq. 
Pour essayer d'apaiser cette préoccupation, la Cour apporta une prétendue solution qui, d'un côté, permettait la consécration de sa doctrine antérieure (les droits fondamentaux font partie des principes généraux du droit communautaire dont la Cour assure le respect; le droit communautaire a une primauté absolue sur le droit national); et, d'un autre côté, rassurait les cours constitutionnelles allemande et italienne. Cette prétendue solution était la doctrine selon laquelle les droits protégés par les Communautés européennes sont tous ceux qui sont aussi protégés par les Constitutions nationales. Il n'existait donc aucun risque de défaut de protection ${ }^{23}$.

Cependant, cette nouvelle construction réveilla les résistances d'autres États membres, notamment le RoyaumeUni qui n'avait, et ne voulait pas avoir, de déclaration des droits dans son ordre interne et qui percevait parfaitement comment cette déclaration indésirable entrait de manière dérobée avec le communautaire. On doit noter que le Royaume-Uni ne possédait pas de déclaration des droits, parce que même s'il avait adhéré à la Convention EDH, son système dualiste n'avait pas transformé la Convention EDH en droit interne. Mais, inversement, au moment de son adhésion aux Communautés, le Royaume-Uni avait transformé tout le droit communautaire en droit interne, considérant qu'il n'introduisait aucune déclaration des droits. C'est pour cela que la construction présentée par la Cour de justice alerta les dirigeants britanniques.

\section{Une délicate incorporation des droits fondamentaux dans les traités européens}

$\mathrm{Au}$ sein des Communautés européennes chacun était conscient de la fragilité de la construction jurisprudentielle élaborée par la Cour de justice. Ainsi, l’on a essayé d'incorporer la reconnaissance des droits fondamentaux dans le texte des traités ${ }^{24}$. Cependant, cette tâche ne fut pas facile et elle présente aujourd'hui encore des problèmes auxquels nous ferons référence ci-après. Ce processus d'incorporation des droits fondamentaux aux traités communautaires a connu plusieurs étapes.
L'Acte unique européen (AUE) ${ }^{25}$, signé à Luxembourg le 17 février 1986, constitue le premier essai de donner une base textuelle dans les traités à la reconnaissance des droits fondamentaux ${ }^{26}$. Ce texte fut le premier pas juridique et positif vers une union politique dans le cadre communautaire. Dans les discussions qui avaient abouti au traité subsistaient encore les confrontations entre les positions des partisans et adversaires de l'inclusion des droits fondamentaux dans les traités. On parviendra finalement à une solution de compromis qui consistait à introduire une mention aux droits fondamentaux dans le préambule du traité de la CEE aux paragraphes 3 et 5 . Ainsi, les droits fondamentaux figuraient pour la première fois dans les traités, mais puisqu'ils se trouvaient dans le préambule et non dans le dispositif, ils ne pouvaient pas déployer une efficacité juridique.

La deuxième étape de ce processus est constituée par le traité de l'Union européenne (TUE) ${ }^{27}$ signé dans la cité néerlandaise de Maastricht le 7 février 1992. Ce traité qui ouvrait un nouveau processus «politique» d'union européenne devait inclure des références aux droits fondamentaux. On allait encore voir se produire l'affrontement entre les positions «européistes» et les positions "étatistes", aboutissant tout comme dans le passé à un compromis ${ }^{28}$. À cette occasion, la mention des droits fondamentaux ne se fit pas seulement dans le préambule (comme c'était le cas dans l'AUE), mais aussi dans les articles du dispositif. Or, ces articles furent expressément soustraits de la compétence de la Cour de justice. De cette façon on arriva, en quelque sorte, au même compromis atteint par l'AUE, même s'il apparaît initialement différent.

Cette insuffisance ne fut pas comblée avec le traité d'Amsterdam de $1997^{29}$ qui n'ajouta rien aux clauses générales déjà contenues dans le TUE, à l'exception des références aux droits sociaux introduits dans le préambule.

Le traité de Nice de $2001^{30}$ fut encore plus timide en la matière et son unique contribution est la réforme du système de vigilance et des sanctions pour les États de

23. Arrêt CJCE, 14 mai 1974, J. Nold, Kohlen und Baustoffgrosshandlung c. Commission des Communautés européennes, C-4/73, Recueil, p. 491 sq. Cet arrêt est postérieur à l'arrêt Frontini et seulement de quelques jours antérieur à l'ordonnance Solange. Cependant, il semble clair que la Cour de justice savait que la Cour constitutionnelle fédérale allemande s'était saisie de l'affaire Solange et était en train d'adopter une position très dure.

24. Voir C. Ruiz-Miguel, «El largo y tortuoso camino... », p. 38 sq.

25. JOCE, L169, 29 juin 1986

26. La Grèce, l'Italie et le Danemark ne l’ont signé que le 28 février.

27. JOCE, C191, 29 juillet 1992.

28. Ce compromis était instable en tant que tel comme le montre le processus de réforme continu des traités ouvert par Maastricht. Il faut se rappeler que même si la signature est datée de février 1992, la procédure d'adhésion fut longue et pénible, avec le rejet par le peuple danois exprimé par référendum. Cette instabilité qui était fondée sur l'impossibilité d'arriver à un consensus dans l'Europe de l'époque (avec alors douze membres) eut une double conséquence. Primo, le déclenchement des «coopérations renforcées» dans quelques domaines essentiels, comme l’Union monétaire ou la Charte sociale des droits des travailleurs. Secundo, le renforcement du discours sur "l'Europe des citoyens", fondement lui-même des programmes et législations concernant la transparence informative des institutions et organes communautaires, depuis le Conseil européen de Birmingham en décembre 1992 (c'est-à-dire, au moment du débat sur la ratification de la première version du traité de l'Union européenne). Voir E. F. Pérez Carrillo, «La transparencia en el funcionamiento de la Unión Europea: el acceso público a los documentos de sus instituciones y órganos", Revista Vasca de Administración Pública, nº 56, 2000, p. 349 sq., spéc. p. 353.

29. JOCE, C340, 10 novembre 1997. Sur les carences du traité d'Amsterdam pour clore les questions ouvertes par Maastricht, voir E. F. Pérez Carrillo, «El Tratado de Ámsterdam. Entre le mercado de los quince y la Europa del siglo XXI», Noticias de la Unión europea, n 186, 2000, p. 171-179.

30. JOCE, C80, 10 mars 2001. Nice est considérée « comme une nouvelle étape dans le processus ouvert par Maastricht [...] et une étape sans vocation de permanence» (E. F. Pérez Carrillo, «El Tratado de Niza, entre la consolidación de la Unión de Maastricht y el debate sobre el futuro de Europa", Revista de estudios europeos, $\mathrm{n}^{\circ} 27,2001$, p. 90). 
l'Union qui violent gravement les droits fondamentaux, compte tenu de la désastreuse expérience des sanctions infligées à l'Autriche en janvier 2000.

Le traité établissant la soi-disant "Constitution» européenne de 2004 introduit une confusion dans ce domaine. Cette «Constitution» incorporait la Charte des droits fondamentaux (CDF), ce qui générait deux ordres de problèmes. D’un côté, elle n'établissait pas un seul statut juridique pour les droits fondamentaux, mais plutôt une multiplicité de régimes juridiques, tous valables, ce qui pouvait conduire à un chevauchement dans leur application. De l'autre côté, elle était ambiguë dans la détermination de la force normative des droits des Constitutions étatiques «au niveau européen ${ }^{31}$.

À l'issue de ce processus, les droits fondamentaux dans l'ordre communautaire (donc sans les droits reconnus dans le traité CEE) sont des normes de droit objectif depuis le traité de Maastricht de 1992, mais non des droits subjectifs dont la CJUE pourrait assurer la protection. De plus, étant donné que l'UE n'était pas partie à la Convention EDH, la CJUE ne pouvait pas être saisie des éventuelles infractions aux droits fondamentaux par des actes de l'UE qui ne pouvaient pas davantage être révisés par la Cour EDH.

Le traité de Lisbonne de 2007 (en vigueur depuis décembre 2009) a essayé de surmonter les difficultés que la «Constitution» européenne de 2004 avait posées à ce sujet.

\section{Les essais de convergence dans le domaine des droits de l'homme entre le Conseil de l'Europe et les Communautés européennes/ Union européenne et la réticence de la Cour de justice de l'Union européenne}

À partir du moment où la Communauté européenne a souhaité incorporer les droits fondamentaux dans son ordre juridique, s'est posé le problème de l'articulation de cette éventuelle reconnaissance et protection des droits fondamentaux avec le système créé par le CoE qui apparaît de plus en plus consolidé.

\section{A. Les interférences entre les deux systèmes}

Les six États de la Communauté étaient conscients que la marche vers l'union politique interférait avec le processus initié par le CoE. C'est pourquoi ils avaient prévu un mécanisme d'articulation entre les deux initiatives. C'est le sens de l'article 45 du projet de SCE. Cette importante disposition, primo, prévoyait que tout citoyen pourrait saisir la Cour de la Communauté pour la défense de ses droits fondamentaux ${ }^{32}$; et, secundo, prévoyait un mécanisme pour essayer de concilier la CJCE avec la Cour EDH. Ce mécanisme précisait la concurrence de trois éléments: d'abord, que devant la Cour EDH serait posée une «question de principe relative à l'interprétation ou à l'étendue des obligations découlant de ladite Convention »; ensuite, que la résolution de la Cour EDH intéressait de ce fait « toutes les parties à cette dernière» (y compris les «six»); et enfin, que la question entraînait, le cas échéant, l'obligation pour la Cour de la Communauté de «surseoir à statuer jusqu'à la solution de cette question par les instances établies par la Convention de sauvegarde des droits de l'homme et des libertés fondamentales ${ }^{33}$. De cette façon on parvenait à une convergence entre les deux processus d'unité européenne même si la Communauté européenne n'était pas partie à la Convention EDH. On permettait par ailleurs que les éventuelles victimes des violations des droits fondamentaux puissent saisir la CJCE pour connaître des mesures présumées violatrices.

\section{B. Une prise en compte jurisprudentielle de la Convention européenne des droits de l'homme}

Cependant, comme nous l'avons déjà dit, le SCE n'aboutit pas. L'échec de la CED eut comme conséquence le classement du projet de SCE. Cela signifie que les Communautés européennes poursuivaient un chemin différent de celui de la voie tracée par le $\mathrm{CoE}$ (qui avait réussi à approuver la Convention EDH avec pour conséquence la création de la Cour EDH).

Dans ce contexte, lorsque la CJCE entreprit de reconnaître des «droits fondamentaux» entendus comme des "principes généraux» du droit communautaire, elle ne fit aucune allusion à la Convention EDH. Mais très tôt elle dut y faire référence pour montrer que la sauvegarde de ces droits tirait son inspiration non seulement des Constitutions des États membres des Communautés, mais aussi des traités internationaux auxquels ces États membres avaient adhéré (une allusion indirecte à la Convention EDH) ${ }^{34}$. Un peu plus tard, la Cour de justice fit une allusion directe à la Convention EDH comme critère d'identification des droits susceptibles de protection dans l'ordre communautaire ${ }^{35}$.

31. Voir C. Ruiz-Miguel, «Los derechos fundamentales de los ciudadanos europeos», in Comentarios a la Constitución Europea, E. Álvarez Conde, V. Garrido Mayol (dir.), Valence, Tirant lo Blanch, 2004, t. II, p. 163-188.

32. «Tout différend résultant d'une décision ou d'une mesure quelconque prise par l'une des institutions de la Communauté, qui touche aux droits reconnus par la Convention de sauvegarde des droits de l'homme et des libertés fondamentales, est déféré à la Cour» (art. 45.1 SCE).

33. "Après l'entrée en vigueur des procédures juridictionnelles prévues par la Convention de sauvegarde des droits de l'homme et des libertés fondamentales, tout différend posant une question de principe relative à l'interprétation ou à l'étendue des obligations découlant de ladite Convention et intéressant de ce fait toutes les parties à cette dernière, entraîne, le cas échéant, l'obligation pour la Cour de surseoir à statuer jusqu'à la solution de cette question par les instances établies par la Convention de sauvegarde des droits de l'homme et des libertés fondamentales» (art. 45.3 SCE).

34. Arrêt CJCE, 14 mai 1974, J. Nold, Kohlen und Baustoffgrosshandlung c. Commission des Communautés européennes.

35. Arrêt CJCE, 13 décembre 1979, Liselotte Hauer c. Land Rheinland-Pfalz, C-44/79, Recueil, p. 3727 sq. 


\section{L'exigence d'une compétence de l'Union en matière de droits fondamentaux}

Dans le sillage de la Cour, le traité sur l'Union européenne de 1992 constitua une étape forte vers une convergence entre l'UE et le CoE, lorsqu'on établit, dans l'article F.2 dudit traité que

L'Union respecte les droits fondamentaux, tels qu'ils sont garantis par la Convention européenne de sauvegarde des droits de l'homme et des libertés fondamentales, signée à Rome le 4 novembre 1950, et tels qu'ils résultent des traditions constitutionnelles communes aux États membres, en tant que principes généraux du droit communautaire.

Dans le «projet de Constitution» approuvé par le Parlement européen en 1994, l'UE ne présente toujours pas de définition du chemin à suivre. On introduit une clause sur les droits de l'homme qui fait référence à la Convention EDH (suivant le modèle du traité de 1992, mais avec quelques changements):

L'Union respecte les droits fondamentaux tels qu'ils sont garantis par la Convention de sauvegarde des droits de l'homme et des libertés fondamentales et par les autres instruments internationaux applicables et tels qu'ils résultent des principes constitutionnels communs aux États membres ${ }^{36}$.

Il faut noter, d'abord, que d'après le texte de cet article l'UE s'engageait à respecter les droits de l'homme pas seulement tels que garantis par la Convention EDH et les «principes constitutionnels communs aux États membres», mais aussi tels que garantis par d' «autres instruments internationaux applicables». Ensuite, il faut noter que dans ce projet de Constitution de l'UE on trouvait un chapitre VIII portant le titre «Droits de l'homme garantis par l'Union» où, pour la première fois, on proposait une charte autonome des droits fondamentaux de l'UE différente de la Convention EDH. Mais ce projet de Constitution fut laissé de côté.

C'est dans ce contexte qu'en 1994 la Commission européenne demanda à la CJUE l'émission d'un avis consultatif sur l'éventuelle adhésion de l'UE à la Convention EDH. L'avis de la Cour, rendu deux ans plus tard, avorta le processus de rapprochement entre l'UE et la Convention $\mathrm{EDH}^{37}$, très probablement en raison de la jalousie de la CJUE. La Cour argumente avec une rigueur qui, si elle avait été auparavant employée par la Cour ellemême, l'aurait amenée à désavouer sa propre construction sur les droits fondamentaux comme faisant partie des "principes généraux» du droit communautaire.
Dans cet avis la Cour fut confrontée à ses propres contradictions: ou bien les Communautés européennes avaient des compétences dans le domaine des droits fondamentaux et donc pouvaient adhérer à la Convention $\mathrm{EDH}$, ou bien les Communautés ne disposaient pas de ces compétences et donc toute sa propre doctrine sur la question se voyait désavouée; ou bien le droit communautaire était «autonome» et avec une "primauté» absolue sur tout autre ordre légal, ou bien le droit communautaire constituait un ordre supérieur, ou bien on devrait reconnaitre un système des droits fondamentaux (la Convention EDH) non élaboré par les Communautés et au-dessus du droit des Communautés. Au final, la Cour établit que la Communauté «ne dispose que de compétences d'attribution ", c'est-à-dire de celles que le traité attribue lui-même sans qu'il soit possible pour la Communauté de s'attribuer des compétences à soi-même sans une préalable modification du traité. À la lumière de ces prémisses, la conclusion était claire: ce n'est que si les droits fondamentaux constituent une «compétence» de la Communauté, qu'il sera possible d'adhérer à la Convention EDH. La Cour en conclut donc qu'

[...] en l'état actuel du droit communautaire, la Communauté n'a pas compétence pour adhérer à la convention européenne de sauvegarde des droits de l'homme et des libertés fondamentales ${ }^{38}$.

Cela signifiait que la clause des droits de l'homme incorporée dans l'article F du TUE était insuffisante.

\section{Un blocage persistant}

Le traité de Lisbonne de 2007 semblait avoir apporté la solution idoine pour résoudre le problème. D'abord, la rédaction de l'article 6 du traité de l'Union européenne selon lequel

Les droits fondamentaux, tels qu'ils sont garantis par la Convention européenne de sauvegarde des droits de l'Homme et des libertés fondamentales et tels qu'ils résultent des traditions constitutionnelles communes aux États membres, font partie du droit de l'Union en tant que principes généraux ${ }^{39}$.

Ensuite, la disposition qui éclipse la CDF en prévoyant que «Les dispositions de la Charte n'étendent en aucune manière les compétences de l'Union telles que définies dans les traités ${ }^{40}$ et que

L’Union adhère à la Convention européenne de sauvegarde des droits de l'Homme et des libertés fondamentales. Cette adhésion ne modifie pas les compétences de l'Union telles qu'elles sont définies dans les traités ${ }^{41}$.

36. Art. 7 du projet de Constitution de l’Union européenne, annexe à la résolution sur la Constitution de l'Union européenne, approuvée par le Parlement européen le 10 février 1994, JOCE, n ${ }^{\circ}$ C 61, 28 février 1994, p. 15 sq.

37. CJUE, avis consultatif sur l'adhésion de la Communauté à la convention de sauvegarde des droits de l'homme et des libertés fondamentales, avis 2/94, 28 mars 1996, Recueil, t. I, p. 1759 sq.

38. Ibid.

39. Art. 6.3 TUE.

40. Art. 6.1 TUE.

41. Art. 6.2 TUE. 
Cependant, huit ans après la signature et six ans après l'entrée en vigueur du traité de Lisbonne, l'UE n'a pas adhéré à la Convention EDH et il semble que cette possibilité est plus lointaine que jamais après l'avis consultatif de la CJUE $2 / 13^{42}$. Ce blocage est encore plus difficile à justifier étant donné deux circonstances: primo, que tous les États membres de l'UE ont adhéré à la Convention EDH; et secundo, que même le texte de la Convention EDH fut amendé avec le protocole $n^{\circ} 14$ (en vigueur depuis le $1^{\text {er }}$ juin 2010) pour introduire un nouvel article 59.2 possédant cette teneur: «L'Union européenne peut adhérer à la présente Convention».

\section{La Charte des droits fondamentaux: de l'essai de se passer du Conseil de l'Europe à la reconnaissance réticente de sa suprématie}

\section{A. La rédaction d'une charte inutile}

Dans ce contexte il faut relever le rôle de la CDF. La genèse de ce texte est très controversée. Lors du sommet de Cologne, en juin 1999, le Conseil européen a estimé

[...] qu'à ce stade du développement de l'Union européenne il conviendrait de réunir les droits fondamentaux en vigueur au niveau de l'Union dans une charte de manière à leur donner une plus grande visibilité ${ }^{43}$.

Dans une annexe à la déclaration de la présidence on disait que «La charte doit en outre contenir les droits fondamentaux réservés aux citoyens de l'Union ${ }^{44}$. L'objectif initial de la CDF était donc de procéder à un recueil de ces droits subjectifs qui avaient été reconnus dans la législation communautaire (aussi bien dans le droit primaire que dans le droit dérivé). Il est vrai que les textes de la déclaration finale et de l'annexe présentaient quelques divergences, notamment sur le nombre et le caractère des droits inclus dans la charte. Si la déclaration parle des «droits fondamentaux en vigueur au niveau de l'Union ", l'annexe parle plutôt d'inclure certains droits qui, très clairement, n'étaient pas en vigueur au niveau de l'Union ${ }^{45}$. Le mandat du sommet de Cologne semblait autoriser la possibilité, déjà posée par le projet de Constitution de l'UE de 1994, que l'UE dispose de sa propre "déclaration des droits» semblable à celle des États.
Cependant, la convention réunie pour élaborer ce texte, présidée par Valéry Giscard d'Estaing, agit ultra vires, en retenant le texte de l'annexe de Cologne tout en laissant de côté le texte de la déclaration principale. D’abord, au lieu de faire un recueil des droits «applicables» au niveau communautaire comme le demandait la déclaration présidentielle, elle a travaillé en vue de rédiger un catalogue des droits "désirables» (comme le suggérait l'annexe, avec une rédaction, à mon avis, inconsistante de la déclaration présidentielle). Ensuite, au lieu de faire l'état des lieux des droits «réservés aux citoyens de l'Union», la convention rédigea un catalogue des droits attribuables aux êtres humains, avec une prétension d'universalité qui allait au-delà de l'objectif initial. L'ambition idéaliste de la CDF, fondée sur une lecture biaisée des textes du sommet de Cologne, se trouve à la racine de son échec d'un point de vue réaliste.

La rédaction d'un texte qui allait au-delà du mandat de la déclaration présidentielle faisait de la CDF un texte inutile. Pour cette raison, lorsque les chefs d'État et de gouvernement se sont réunis lors du sommet de Nice pour décider quoi faire de ce texte, ils ont pris la décision la plus conforme avec la réalité et les usages diplomatiques : la réalité imposait que le texte ne puisse pas bénéficier d'une force juridique positive; les usages diplomatiques suggéraient une sortie honorable pour les membres de la convention qui avaient rédigé le texte. En conclusion, la CDF fut «proclamée» dans le sommet de Nice... mais elle ne fut pas incorporée aux traités.

\section{B. Une concurrence impossible de la Convention européenne des droits de l'homme}

La convention, créée pour accomplir le mandat du sommet de Nice, avait tenté de réussir quelque chose d'irréalisable en dépassant la tâche qui lui avait été assignée: créer une déclaration européenne des droits de l'homme concurrençant la Convention EDH. Plusieurs raisons expliquent cet échec prévisible. Tout d'abord, le passage du temps avait consolidé le mécanisme de protection des droits de l'homme créé par le CoE; ensuite, l'incorporation dans la CDF des droits de l'homme qui étaient très loin des activités de l'UE (tel que le droit à la vie) n'était pas réaliste en relation avec les compétences et la nature de l'UE; finalement, parce que la CDF était techniquement très inférieure à la Convention EDH.

42. CJUE, Ass. plén., avis 2/13, 18 décembre 2014.

43. Conseil européen de Cologne, 3 et 4 juin 1999, conclusions de la présidence, $\$$ 44, en ligne: http://www.europarl.europa.eu/summits/kol1_fr.htm.

44. Ibid., "Annexe IV. Décision du Conseil européen concernant l'élaboration d'une charte des droits fondamentaux de l'union européenne", en ligne: http://www.europarl.europa.eu/summits/kol2_fr.htm\#an4.

45. «Le Conseil européen est d'avis que cette charte doit contenir les droits de liberté et d'égalité, ainsi que les droits de procédure tels que garantis par la Convention européenne de sauvegarde des droits de l'homme et des libertés fondamentales et tels qu'ils résultent des traditions constitutionnelles communes des États membres, en tant que principes généraux du droit communautaire. La charte doit en outre contenir les droits fondamentaux réservés aux citoyens de l'Union. Dans l'élaboration de la charte, il faudra par ailleurs prendre en considération des droits économiques et sociaux tels qu'énoncés dans la Charte sociale européenne et dans la Charte communautaire des droits sociaux fondamentaux des travailleurs (article 136 TCE [Traité instituant la Communauté européenne]) dans la mesure où ils ne justifient pas uniquement des objectifs pour l'action de l'Union» (ibid.) 
La soi-disant «Constitution» européenne de 2004 fut un essai plutôt irréfléchi de donner une nature juridique à la CDF. Le traité de Lisbonne aurait dû reprendre l'influence du mandat du sommet de Cologne de 1999, mais une telle démarche aurait pu signifier un désaveu du travail accompli par la convention présidée par Valéry Giscard d'Estaing. On essaya donc, comme un moindre mal, de corriger la situation créée par la soi-disant «Constitution » européenne sans désavouer les efforts accomplis. Ces amendements se trouvent principalement dans le titre VII de la CDF («Dispositions générales régissant l'interprétation et l'application de la Charte»).

Au vu des considérations précédentes, il faut souligner quelques observations.

Primo, la CDF n'est pas comparable à la Convention $\mathrm{EDH}$, parce qu'elle n'a pas une portée générale: d'un côté, «Les dispositions de la Charte n'étendent en aucune manière les compétences de l'Union telles que définies dans les traités $»^{46}$; mais de l'autre côté, on affirme que la CDF du 7 décembre 2000, «telle qu'adoptée le 12 décembre 2007 à Strasbourg, [...] a la même valeur juridique que les traités ${ }^{47}$. L'interprétation systématique de ces préceptes nous révèle que la CDF s'applique seulement aux politiques communautaires. Contrairement à ce qui se passe avec la Convention EDH, qui établit des droits applicables de façon générale dans tous les contextes, la CDF établit seulement des droits invocables là où il $\mathrm{y}$ a des relations régies par le droit communautaire et seulement là.

Secundo, précisément parce que la CDF est uniquement applicable aux relations régies par le droit communautaire, on peut mettre en question ce texte lorsqu'il inclut des droits dont il est très difficile de croire qu'ils peuvent être appliqués à des situations régies par le droit communautaire. On peut penser au droit à la vie et à l'interdiction de la peine de mort (art. 2 CDF), à l'interdiction de la torture (art. 4 CDF), de l'esclavage (art. 5 $\mathrm{CDF}$ ), au droit de se marier (art. $9 \mathrm{CDF}$ ), parmi quelques autres. La même position peut être suivie si on parle des droits de «solidarité» qui ne peuvent pas signifier une «consolidation» des «droits fondamentaux en vigueur au niveau de l'Union». Il est alors difficile d'expliquer pourquoi ces «droits» sont encore présents dans la CDF, créant une fausse expectative.

Les observations précédentes sont encore plus claires si on examine les «Explications relatives à la Charte des droits fondamentaux» que le traité de Lisbonne précise ${ }^{48}$. Dans ces «Explications» on constate que certains droits de la Charte qui n'existent pas dans le droit communautaire n'existent qu'en référence à la Convention EDH et à la jurisprudence de la Cour EDH sur la Convention.

\section{Une alternative non viable}

Pour toutes les raisons précédemment exposées, on peut dire que la CDF considérée comme une «alternative» à la Convention EDH n'est pas viable. Pour cette raison la teneur du nouvel article 6.2 du TUE après le traité de Lisbonne était tout à fait raisonnable:

L'Union adhère à la Convention européenne de sauvegarde des droits de l'Homme et des libertés fondamentales. Cette adhésion ne modifie pas les compétences de l'Union telles qu'elles sont définies dans les traités.

Mais on ne peut pas contester que le Protocole $n^{\circ} 8$ au traité de Lisbonne ${ }^{49}$, qui précise que l'éventuel accord d'adhésion à la Convention $\mathrm{EDH}$ «doit refléter la nécessité de préserver les caractéristiques spécifiques de l'Union et du droit de l'Union ${ }^{50}$ et que l'adhésion de l'Union ne doit affecter ni les compétences de l'Union ni les attributions de ses institutions ${ }^{51}$, révèle une forte méfiance. Quelle est la portée exacte de l'expression «préserver» les «caractéristiques spécifiques» du «droit de l'Union»? Ceci n'était pas très clair au moment de l'approbation du traité de Lisbonne... et n'est pas plus clair après l'adoption de l'avis consultatif du 18 décembre 2014. Tous ces éléments semblent signifier la persistance de l'ancienne jalousie des organes de l'UE, notamment de la CJUE, envers le $\mathrm{CoE}$, et notamment envers la Cour EDH. Il est difficile de trouver des raisons objectives pour expliquer pourquoi la cession des compétences (soit à l'UE, soit au CoE) ne fut traumatique pour aucun État européen, mais fut si problématique pour l'UE composée de ces mêmes États. La démarche tactique d'élaborer la CDF comme sa propre déclaration des droits pour éviter que l'UE se soumette à un contrôle externe a échoué, et il serait bon que l'UE accepte cette réalité.

\section{Une convergence improbable: les difficultés et obstacles issus de l'avis de la Cour de justice de l'Union européenne du 18 décembre 2014}

La convergence entre les deux voies d'unification politique européenne fondée sur un statut commun concernant

46. Art. 6.1.2 TUE et, dans un sens identique, art. 51.2 CDF: «La présente Charte n'étend pas le champ d'application du droit de l'Union au-delà des compétences de l'Union, ni ne crée aucune compétence ni aucune tâche nouvelle pour l'Union et ne modifie pas les compétences et tâches définies dans les traités".

47. Art. 6.1.1 TUE

48. «Les explications élaborées en vue de guider l'interprétation de la présente Charte sont dûment prises en considération par les juridictions de l'Union et des États membres» (art. 52.7 CDF).

49. Protocole $\mathrm{n}^{\circ} 8$ relatif à l'article $6, \$ 2$, du traité sur l'Union européenne sur l'adhésion de l'Union à la Convention européenne de sauvegarde des droits de l'homme et des libertés fondamentales.

50. Art. 1 du Protocole $n^{\circ} 8$ au traité de Lisbonne.

51. Art. 2 du Protocole $n^{\circ} 8$ au traité de Lisbonne. 
les droits fondamentaux des Européens s'est heurtée à plusieurs difficultés et obstacles. Des difficultés sont nées de la part de quelques (ou quelque) État(s) membre(s) de l'UE où existent des tendances politiques plutôt critiques envers la Cour EDH. Existent aussi les problèmes occasionnés par les conséquences pour l'UE de l'interprétation faite par la Cour EDH du texte de la Convention EDH. Et à tout cela s'est ajoutée une difficulté interne à l'UE avec l'avis consultatif de la CJUE 2/13 concernant l'éventuelle adhésion de l'UE à la Convention EDH.

\section{A. Des implications pour le Royaume-Uni}

L'adhésion de l'UE à la Convention EDH aurait des implications dans le débat interne du Royaume-Uni, qui est l'État où s'est posée le plus ouvertement la question de se retirer de la Convention EDH. Dans le système dualiste britannique de relation entre le droit interne et le droit international, le droit communautaire fait partie du droit interne depuis l'adhésion du Royaume-Uni aux Communautés en 1972, mais la Convention EDH ne fut transformée en droit interne qu'en 1998 avec l'approbation de la loi sur les droits de l'homme (Human Rights $A(t)$. Mais il existe aujourd'hui un intense débat politique au Royaume-Uni sur la possibilité d'abroger cette loi des droits de l'homme ${ }^{52}$. Cette possibilité pourrait cependant être entravée si l'UE adhère à la Convention EDH, puisque cette éventualité aboutirait à ce que la vigueur interne de la Convention EDH revienne au Royaume-Uni de manière indirecte. Étant donné la force expansive du droit communautaire, il y a fort à parier que dans de nombreux cas, la Convention EDH serait applicable en droit interne britannique... ce que certains courants politiques britanniques veulent justement éviter. Il existe donc une indéniable volonté du Royaume-Uni pour que l'UE n'adhère pas à la Convention EDH.

\section{B. Un imparfait « amparo communautaire européen "}

Le traité de Lisbonne a ouvert la porte à une convergence entre l'UE et le CoE concernant les normes qui définissent de façon générale les droits fondamentaux des Européens à travers l'adhésion de l'UE à la Convention EDH. Dès lors ce texte deviendra le vrai critère de définition des droits fondamentaux de l'UE. Mais les prévisions du traité de Lisbonne envisagent également la saisie de la Cour $\mathrm{EDH}$ aux fins de vérifier les atteintes portées par les actes communautaires auxdits droits.
Cependant, le retard et la réticence de l'UE à l'adhésion à la Convention EDH posent la question de la protection juridictionnelle des droits fondamentaux dans l'UE. Si nous employons le concept d' "amparo» dans un sens strict en tant que protection juridictionnelle spécifique des droits fondamentaux, il est tout à fait évident qu'il n'y a pas d'amparo en droit communautaire. Le projet de Constitution de l'UE de 1994 accordait à la Cour de justice une compétence spécifique pour juger les «violations des droits de l'homme». L'article 38 dudit projet de 1994 précisait que

La Cour de Justice est compétente pour se prononcer sur tout recours introduit par un particulier visant à établir la violation par l'Union d'un droit de l'homme garanti par la Constitution.

Une loi constitutionnelle détermine les conditions d'introduction de ce recours et les sanctions que la Cour de Justice peut décider.

Il s'agissait d'introduire un «amparo communautaire européen ». Cependant, comme on l'a précédemment évoqué, ce projet de Constitution fut laissé de côté et plusieurs de ses innovations, telles que celle-ci, ne furent pas prises en compte ultérieurement.

Depuis l'entrée en vigueur du traité de Lisbonne on peut dire qu'il existe un «amparo » imparfait au sein de l'UE, mais uniquement si on utilise le concept d' «amparo» au sens large, renvoyant tout simplement à la possibilité de défendre devant une juridiction ses droits fondamentaux, sans suivre une procédure spécifique. En effet, l'article 263.4 du traité sur le fonctionnement de l'Union européenne (TFUE), dans la teneur que lui avait donnée le traité de Lisbonne (signé en 2007, en vigueur depuis 2009) prévoit que

Toute personne physique ou morale peut former, dans les conditions prévues aux premier et deuxième alinéas, un recours contre les actes dont elle est le destinataire ou qui la concernent directement et individuellement, ainsi que contre les actes réglementaires qui la concernent directement et qui ne comportent pas de mesures d'exécution.

La CJUE eut l'occasion d'interpréter le précepte du traité CEE qui est à l'origine du présent article 263.4 TFUE ${ }^{53}$. Dans l'une des plus importantes affaires où la Cour s'est prononcée sur cette question, elle a clairement précisé que

Dès lors les particuliers doivent pouvoir bénéficier d'une protection juridictionnelle effective des droits qu'ils tirent de l'ordre juridique communautaire, le droit à une telle protection faisant partie des principes généraux de droit qui découlent des traditions constitutionnelles communes aux États membres. Ce droit a également été consacré par les articles 6 et 13 de la convention européenne de sauvegarde des droits de l'homme et des libertés fondamentales ${ }^{54}$.

52. Un groupe de députés britanniques a proposé d'abroger la Human Rights Act et de lui substituer une British Rights Act, accompagnée du retrait du Royaume-Uni de la Convention EDH. Voir «British Bill of Rights and Withdrawal from the European Convention on Human Rights Bill», $1^{\text {re }}$ lecture, 7 juillet $2014 ; 2^{\mathrm{e}}$ lecture, 20 mars 2015.

53. Art. 173.2 (postérieurement numéroté art. 230.4) du traité CEE: «Toute personne physique ou morale peut former, dans les mêmes conditions, un recours contre les décisions dont elle est le destinataire et contre les décisions qui, bien que prises sous l'apparence d'un règlement ou d'une décision adressée à une autre personne, la concernent directement et individuellement».

54. Arrêt de la CJUE, 25 juillet 2002, Unión de Pequeños Agricultores c. Conseil de l’Union européenne, C-50/oo P, § 39. 
Or, cette protection agit seulement envers les normes qui sont matériellement singulières, même si elles revêtent la forme de normes générales:

Cependant, un acte de portée générale tel qu'un règlement peut, dans certaines circonstances, concerner individuellement certaines personnes physiques ou morales, revêtant dès lors un caractère décisionnel à leur égard ${ }^{55}$.

Voici donc l'une des différences entre la protection juridictionnelle des droits fondamentaux dans le cadre de l'UE et dans le cadre de la Convention EDH du CoE. Ce n'est pas seulement parce que la Convention EDH prévoit une procédure spécifique pour la protection des droits fondamentaux, contrairement à ce qui s'est passé avec les traités communautaires. La différence peut-être la plus importante parmi les deux systèmes est que lorsque la Cour EDH a accepté d'examiner (certes en faisant violence au texte de la Convention EDH) des demandes individuelles contre des normes formelles et matériellement générales ${ }^{56}$, la CJUE n'a pas accepté les demandes individuelles contre des normes matériellement générales ${ }^{57}$. Cette différence entre les critères de la Cour EDH et de la CJUE pour déterminer la possibilité de contestation des normes matériellement générales pourrait être un des obstacles fondamentaux qui empêchent la convergence entre l'UE et le CoE telle que prévue par le traité de Lisbonne.

\section{Une pertinente demande d'avis consultatif}

Le protocole $\mathrm{n}^{\circ} 14$ à la Convention EDH qui introduit un nouvel article 59.2 («L'Union européenne peut adhérer à la présente Convention») est entré en vigueur le $1^{\text {er }}$ juin 2010, quelques mois après l'entrée en vigueur du traité de Lisbonne en décembre 2009 et quelques jours avant l'introduction du nouvel article 59.2 de la Convention EDH.

Lors de leur $1085^{\mathrm{e}}$ réunion (26 mai 2010), les délégués des États membres du CoE avaient adopté un mandat occasionnel chargeant le Comité directeur pour les droits de l'homme (CDDH) d'élaborer, au plus tard le 30 juin 2011 (en coopération avec les représentants de l'UE), un instrument juridique établissant les modalités d'adhésion de l'UE à la Convention EDH. À la fin des négociations, les deux parties étaient parvenues à un accord sur un ensemble d'instruments juridiques pour faciliter l'adhésion de l'UE à la Convention EDH. Ces instruments consistent en 1) un projet d'accord sur l'adhésion de l'UE à la Convention $\mathrm{EDH}, 2$ ) un projet de déclaration de l'UE, 3) un projet de règles à ajouter aux règles du Comité des ministres pour la surveillance de l'exécution des arrêts et des termes des règlements amiables dans les affaires auxquelles l'UE est partie, 4) un projet de modèle de mémorandum d'accord, et 5) un projet de rapport explicatif à l'accord d'adhésion ${ }^{58}$. Quelques semaines après la présentation de cet accord, la Commission européenne a introduit une demande d'avis consultatif auprès de la CJUE le 4 juillet 2013 en posant cette question:

Est-ce que le projet d'accord portant adhésion de l'Union européenne à la convention de sauvegarde des droits de l'homme et des libertés fondamentales [signée à Rome le 4 novembre 1950] est compatible avec les traités?

La preuve la plus claire de l'intérêt et de la pertinence de la demande d'avis consultatif présentée par la Commission se trouve dans le nombre des parties qui avaient présenté des observations à la Cour, par écrit ou oralement, lors de l'audience: les gouvernements belge, bulgare, tchèque, danois, allemand, estonien, irlandais, grec, espagnol, français, italien, chypriote, letton, lituanien, hongrois, néerlandais, autrichien, polonais, portugais, roumain, slovaque, finlandais, suédois et du Royaume-Uni, ainsi que le Parlement et le Conseil de l'Union. Il faut noter que même si leurs appréciations se distinguent de celles de la Commission sur plusieurs points, l'ensemble des États membres et des institutions susmentionnés concluent, en substance, à la compatibilité du projet d'accord avec les traités.

\section{Les conclusions de l'avis du 18 décembre 2014}

La CJUE a rendu son avis le 18 décembre $2014^{59}$ :

L'accord portant adhésion de l'Union européenne à la Convention européenne de sauvegarde des droits de l'homme et des libertés fondamentales n'est pas compatible avec l'article 6, paragraphe 2, TUE ni avec le protocole $\left(n^{\circ} 8\right)$ relatif à l'article 6, paragraphe 2, du traité sur l'Union européenne sur l'adhésion de l'Union à la Convention européenne de sauvegarde des droits de l'homme et des libertés fondamentales.

Il peut être étonnant que la CJUE arrive à cette conclusion alors que toutes les observations des organes communautaires (Commission, Conseil et Parlement) et des vingt-quatre États membres de l'UE avaient manifesté une opinion tout à fait contraire. L'analyse des cinq arguments de la CJUE pour retenir la non-compatibilité avec les traités communautaires du projet d'accord d'adhésion à la Convention EDH semble effectivement faible.

55. Arrêt de la CJUE, 25 juillet 2002, Unión de Pequeños Agricultores c. Conseil de l’Union européenne, $\$ 36$.

56. Voir C. Ruiz-Miguel, La ejecución de las sentencias del Tribunal Europeo de Derechos Humanos. Un estudio sobre la relación entre el derecho nacional y el internacional, Madrid, Tecnos, 1997.

57. Ordonnance du tribunal, $7^{\mathrm{e}} \mathrm{ch}$. élargie, 6 septembre 2011, Inuit Tapiriit Kanatami et autres c. Parlement européen et Conseil de l'Union européenne, $\mathrm{T}-18 / 10, \$ 44-50$.

58. Les textes dudit accord se trouvent dans le document Cinquième réunion de négociation entre le groupe de négociation ad hoc du CDDH et la Commission européenne sur l'adhésion de l'Union européenne à la Convention européenne des droits de l'homme. Rapport final au CDDH, 10 juin 2013, 47+1(2013)oo8rev2.

59. CJUE, Ass. plén., avis $2 / 13$. 


\section{a) Le projet d'accord}

[...] est susceptible de porter atteinte aux caractéristiques spécifiques et à l'autonomie du droit de l'Union, dans la mesure où il n'assure pas la coordination entre l'article 53 de la CEDH et l'article 53 de la Charte, ne prévient pas le risque d'atteinte au principe de la confiance mutuelle entre les États membres dans le droit de l'Union ${ }^{60}$.

Cet argument pose une des questions clés de l'adhésion de l'UE à la Convention EDH. L'élément central de cet argument est très simple: est-ce qu'avoir un droit européen est plus important que fournir une large protection des droits fondamentaux? La réponse de l'article 53 de la Convention EDH est "non", mais la réponse de la CJUE est «oui». La réponse de la CJUE dans l'affaire Meloni était claire: la préservation de la primauté d'un droit communautaire unifié est plus importante que la possibilité de fournir des protections des droits fondamentaux plus larges que celles offertes par le droit communautaire ${ }^{61}$. Ceci veut tout simplement dire que pour la CJUE la possibilité de se voir désavouée par la Cour EDH est insoutenable si celle-ci donne une interprétation des droits fondamentaux plus large que celle donnée par la CJUE.

\section{b) Le projet d'accord}

[...] est susceptible d'affecter l'article 344 TFUE, dans la mesure où il n'exclut pas la possibilité que des litiges entre les États membres ou entre ces derniers et l'Union, relatifs à l'application de la CEDH dans le champ d'application matériel du droit de l'Union, soient portés devant la Cour EDH ${ }^{62}$.

Cet argument est fallacieux. Lorsque la CJUE dit que le projet d'accord est susceptible d'affecter l'article 344 TFUE («Les États membres s'engagent à ne pas soumettre un différend relatif à l'interprétation ou à l'application des traités à un mode de règlement autre que ceux prévus par ceux-ci»), elle oublie que l'article 6.1 TUE lui-même introduit une exception à l'article 344 TFUE lorsqu'il s'agit de l'interprétation des droits fondamentaux reconnus dans la Convention EDH.

\section{c) Le projet d'accord}

[...] ne prévoit pas des modalités de fonctionnement du mécanisme de codéfendeur [...] qui permettent de préserver les caractéristiques spécifiques de l'Union et de son droit ${ }^{63}$.
La même position est adoptée concernant le mécanisme de codéfendeur. La CJUE rejette la possibilité que la Cour EDH puisse déterminer que seul l'un d'entre le défendeur et le codéfendeur puisse être tenu pour responsable d'une violation de la Convention EDH parce qu'une telle décision pourrait porter préjudice à la répartition des compétences entre l'Union et ses États membres. Or, à partir du moment où le TUE lui-même introduit la possibilité d'adhérer à la Convention EDH, le TUE introduit indéniablement une exception aux règles de répartition des compétences entre l'UE et ses États membres contenues dans d'autres articles des traités.

\section{d) Le projet d'accord}

[...] ne prévoit pas des modalités de fonctionnement du mécanisme $[. .$.$] de la procédure préalable de la Cour qui$ permettent de préserver les caractéristiques spécifiques de l'Union et de son droit ${ }^{64}$.

Dans le cadre des rencontres régulières entre ses délégations, la Convention EDH et la CJUE avaient abordé, à propos de l'éventuelle adhésion de l'UE à la Convention $\mathrm{EDH}$, la question de l'éventuelle implication préalable de la CJUE dans les affaires dans lesquelles l'UE serait codéfenderesse. Le «Projet de rapport explicatif à l'Accord portant adhésion de l'Union européenne à la Convention de sauvegarde des droits de l'homme et des libertés fondamentales » (Annexe $\mathrm{V}$ du projet d'accord) précise que

Les affaires dans lesquelles l'UE peut être codéfendeur ont leur origine dans des requêtes individuelles concernant des actes ou omissions des États membres de l'UE. Le requérant devra en premier lieu épuiser les voies de recours internes disponibles dans les juridictions nationales de l'État membre défendeur. Ces juridictions nationales peuvent, et dans certains cas doivent, saisir la CJUE d'un renvoi préjudiciel portant sur l'interprétation et/ou la validité d'une disposition litigieuse du droit de l'UE (article $267 \mathrm{du}$ TFUE). Puisque les parties à l'affaire ne peuvent, devant les juridictions nationales, que suggérer un tel renvoi, cette procédure ne peut pas être considérée comme une voie de recours à épuiser par le requérant avant de saisir la Cour. Or, s'il n'était pas procédé à un tel renvoi préjudiciel, la Cour serait appelée à se prononcer sur la conformité d'un acte de l'UE avec les droits de l'homme, sans que la

60. Ibid., $\$ 258$ et voir $\$ 179-200$.

61. Arrêt de la CJUE, GC, 26 février 2013 (demande de décision préjudicielle du tribunal constitutionnel, Madrid, Espagne), Stefano Melloni c. Ministerio Fiscal, C-399/11, $\$ 58,59$ et 64 : «58. En effet, cette interprétation de l'article 53 de la Charte porterait atteinte au principe de la primauté du droit de l'Union, en ce qu'elle permettrait à un État membre de faire obstacle à l'application d'actes du droit de l'Union pleinement conformes à la Charte, dès lors qu'ils ne respecteraient pas les droits fondamentaux garantis par la Constitution de cet État. / 59. Il est, en effet, de jurisprudence bien établie qu'en vertu du principe de la primauté du droit de l'Union, qui est une caractéristique essentielle de l'ordre juridique de l'Union [...], le fait pour un État membre d'invoquer des dispositions de droit national, fussent-elles d'ordre constitutionnel, ne saurait affecter l'effet du droit de l'Union sur le territoire de cet État [...]./ [...] 64. Eu égard aux considérations qui précèdent, il convient de répondre à la troisième question que l'article 53 de la Charte doit être interprété en ce sens qu'il ne permet pas à un État membre de subordonner la remise d'une personne condamnée par défaut à la condition que la condamnation puisse être révisée dans l'État membre d'émission, afin d'éviter une atteinte au droit à un procès équitable et aux droits de la défense garantis par sa Constitution».

62. CJUE, Ass. plén., avis $2 / 13, \$ 258$ et voir $\$ 201-214$

63. Ibid., $\$ 258$ et voir $\$ 215-235$.

64. Ibid., $\$ 258$ et voir $\$ 236-257$ 
CJUE ait eu l'occasion de le faire, en statuant, selon les cas, sur la validité d'une disposition du droit dérivé ou sur l'interprétation d'une disposition du droit primaire ${ }^{65}$.

Dans ces observations à la demande d'avis consultatif, la Commission avait suggéré que la procédure de l'implication préalable de la Cour ne devrait pas figurer dans un accord international, mais devrait être arrêtée, de manière autonome, au niveau de l'Union, dès lors qu'elle a pour vocation de régir une procédure interne à cette dernière. Au demeurant, il ne serait pas nécessaire ni même approprié d'insérer cette procédure de l'implication préalable dans les traités. La Commission estimait qu'il était plus approprié que les dispositions posant le principe même d'une procédure d'implication préalable de la Cour et définissant les entités compétentes pour l'engager ainsi que les standards au regard desquels l'examen de la compatibilité devrait être effectué relèvent de la décision du Conseil portant conclusion de l'accord d'adhésion. En ce qui concerne le contenu des dispositions internes régissant la procédure de l'implication préalable de la Cour, tout d'abord, le pouvoir d'engager cette procédure par une demande adressée à la Cour devrait revenir à la Commission et à l'État membre contre lequel la requête devant la Cour EDH est dirigée. En outre, la Cour devrait pouvoir se prononcer avant que l'Union et l'État membre concerné ne prennent position devant la Cour EDH. Ensuite, puisque la procédure présenterait certaines similitudes structurelles avec la procédure de demande préjudicielle, les règles concernant le droit d'y participer devraient être analogues à celles figurant à l'article 23 du statut de la CJUE. Enfin, les exigences de célérité pourraient être satisfaites par l'application de la procédure accélérée visée à l'article 23 bis de ce statut.

Tout cela est une des conséquences de l'absence d'une procédure d' "amparo» communautaire telle que prévue dans l'article 38 du projet de Constitution de $1994^{66}$. En tout cas, il est clair qu'il s'agit d'une question interne du droit communautaire qui ne peut pas justifier une prétendue incompatibilité avec les traités communautaires du projet d'accord d'adhésion à la Convention EDH.

e) Le projet d'accord

[...] méconnaît les caractéristiques spécifiques du droit de l'Union concernant le contrôle juridictionnel des actes, actions ou omissions de l'Union en matière de PESC [politique étrangère et de sécurité commune], dans la mesure où il confie le contrôle juridictionnel de certains de ces actes, actions ou omissions exclusivement à un organe externe à l'Union ${ }^{67}$.
Voici un argument qui semble tout à fait cynique. La CJUE reconnaît que d'après les traités certains actes adoptés dans le cadre de la PESC échappent au contrôle juridictionnel de la CJUE. Pour justifier sa position, la CJUE cite un autre avis selon lequel la CJUE devrait se prononcer sur la compatibilité avec les traités d'une juridiction internationale (la juridiction du brevet européen) chargée d'interpréter et d'appliquer le droit de l'UE dans le domaine des brevets. Or, il y a ici une différence fondamentale parce que la Cour EDH interprète, mais n'applique pas, la Convention EDH (nous reviendrons sur ce sujet) ${ }^{68}$.

Dans la présente affaire, la CJUE, citant l'avis 1/o9, précise que

[...] la compétence pour effectuer un contrôle juridictionnel d'actes, d'actions ou d'omissions de l'Union, y compris au regard des droits fondamentaux, ne saurait être attribuée exclusivement à une juridiction internationale qui se situe en dehors du cadre institutionnel et juridictionnel de l'Union ${ }^{69}$.

Il est étonnant que la CJUE puisse méconnaître les compétences de la Cour EDH. Celle-ci interprète seulement, mais n'applique pas et ne contrôle pas la Convention EDH. Si l'on comprend le contrôle comme le concept qui ajoute une sanction, qui empêche une mesure lors d'un jugement négatif ${ }^{70}$, on peut dire que la juridiction de la Cour EDH n'applique ni ne contrôle pas parce que sa compétence est seulement déclaratoire ${ }^{71}$. En conclusion, le problème n'est peut-être pas que le projet d'accord «méconnaît les caractéristiques spécifiques du droit de l'Union » mais que la CJUE méconnaît les caractéristiques spécifiques du droit de la Convention EDH.

\section{E. Les véritables raisons du rejet}

Si les raisons précédemment analysées ne sont guère convaincantes, il faut considérer quelles peuvent être les véritables raisons du rejet de la CJUE à l'adhésion à la Convention EDH. Deux explications peuvent être avancées : l'une relevant de la Cour EDH, et l'autre de la CJUE.

La première raison est que, contrairement aux prévisions textuelles de la Convention EDH et à une jurisprudence établie, la Cour EDH s'est caractérisée ces dernières années par une dérive de ses arrêts afin de tenter d'élargir la portée de ses jugements pour leur donner une force exécutoire. L'affaire emblématique à ce sujet est probablement l'affaire Del Rio Prada. En l'espèce, la Chambre, comme la Grande Chambre de la Cour EDH avaient rendu un jugement précisant «qu'il incombe à l'État défendeur d'assurer

65. Cinquième réunion de négociation..., «Annexe V. Projet de rapport explicatif à l'Accord portant adhésion de l’Union européenne à la Convention de sauvegarde des droits de l'homme et des libertés fondamentales», $\$ 65$.

66. «La Cour de justice est compétente pour se prononcer sur tout recours introduit par un particulier visant à établir la violation par l'Union d'un droit de l'homme garanti par la Constitution".

67. CJUE, Ass. plén., avis $2 / 13, \$ 258$ et voir $\$ 249-257$.

68. CJUE, Ass. plén., avis 1/o9, 8 mars 2011, $\$ 78$ et 89 .

69. Nous soulignons.

70. S. Galeotti, "Controlli costituzionali », in Enciclopedia del diritto, F. Calasso (dir.), Varèse, Giuffrè, 1962, t. X, p. 319 sq., spéc. p. 321.

71. Voir C. Ruiz-Miguel, La ejecución de las sentencias..., p. 62-71. 
la remise en liberté de la requérante dans les plus brefs délais ${ }^{72}$. La question de la force exécutoire de cet arrêt avait déclenché un fort débat au sein de l'opinion publique et juridique espagnole. La démarche de la Cour EDH a indéniablement provoqué de nombreuses réticences.

Cela explique pourquoi la CJUE avait souligné que l'arrêt de la Cour EDH « est déclaratoire et n'affecte pas la validité des actes en cause de la Partie contractante ${ }^{73}$. Si l'on se rappelle le débat sur le retrait du Royaume-Uni de la Convention EDH, on ne saurait s'étonner de voir que le Royaume-Uni a lui aussi insisté sur le caractère déclaratoire dudit arrêt ${ }^{74}$. Or, une mutation de la nature des arrêts de la Cour EDH provoquée par cette Cour elle-même risque de mettre en question toute la construction. Consciente de ce risque, on peut comprendre que la CJUE se méfie du fait qu'un hypothétique changement des circonstances avalisant une éventuelle adhésion de
l'UE à la Convention EDH (notamment, un retrait du Royaume-Uni de la Convention EDH) puisse accroître le risque que la Cour EDH prenne la voie d'une mutation de la nature déclarative de ses arrêts.

La deuxième raison explicative peut relever de la jalousie de la CJUE envers la Cour EDH comme dernière instance d'interprétation. Cette motivation pourrait être sous-jacente aussi bien dans l'avis 2/13, que dans l'avis 1/94. La force d'attraction des normes de droit fondamental sur nombre de domaines est bien connue. Dans le cas où l'UE adhérerait à la Convention EDH, cette force d'attraction serait en mesure d'absorber les litiges les plus divers sous la rubrique des droits fondamentaux. La possibilité de soumettre ces affaires à la Cour EDH est une invitation à faire de celle-ci la vraie instance ultime du droit européen. Une tentation très forte qui ne peut que générer de fortes résistances. 